University of Wollongong

Research Online

Australian Institute for Innovative Materials -

Papers

Australian Institute for Innovative Materials

$1-1-2014$

Electrically contractile polymers augment right ventricular output in the heart

Arjang Ruhparwar

University Hospital of Heidelberg

Patricia Piontek

University Hospital of Heidelberg

Matthias Ungerer

University Hospital of Heidelberg

Ali Ghodsizad

University Hospital of Heidelberg

Sasan Partovi

University Hospital of Heidelberg

See next page for additional authors

Follow this and additional works at: https://ro.uow.edu.au/aiimpapers

Part of the Engineering Commons, and the Physical Sciences and Mathematics Commons

Research Online is the open access institutional repository for the University of Wollongong. For further information contact the UOW Library: research-pubs@uow.edu.au 


\title{
Electrically contractile polymers augment right ventricular output in the heart
}

\begin{abstract}
Research into the development of artificial heart muscle has been limited to assembly of stem cellderived cardiomyocytes seeded around a matrix, while nonbiological approaches to tissue engineering have rarely been explored. The aim of the study was to apply electrically contractile polymer-based actuators as cardiomyoplasty for positive inotropic support of the right ventricle. Complex trilayer polypyrrole (PPy) bending polymers for high-speed applications were generated. Bending motion occurred directly as a result of electrochemically driven charging and discharging of the PPy layers. In a rat model $(n=5)$, strips of polymers $(3 \times 20 \mathrm{~mm})$ were attached and wrapped around the right ventricle (RV). $\mathrm{RV}$ pressure was continuously monitored invasively by direct RV cannulation. Electrical activation occurred simultaneously with either diastole (in order to evaluate the polymer's stand-alone contraction capacity; group 1) or systole (group 2). In group 1, the pressure generation capacity of the polymers was measured by determining the area under the pressure curve (area under curve, AUC). In group 2, the RV pressure AUC was measured in complexes directly preceding those with polymer contraction and compared to RV pressure complexes with simultaneous polymer contraction. In group 1, the AUC generated by polymer contraction was $2768+/-875 \mathrm{U}$. In group 2 , concomitant polymer contraction significantly increased AUC compared with complexes without polymer support ( $5987+/-1334 \mathrm{U}$ vs. 4318 $+/-691 \mathrm{U}, P \leq 0.01$ ). Electrically contractile polymers are able to significantly augment right ventricular contraction. This approach may open new perspectives for myocardial tissue engineering, possibly in combination with fetal or embryonic stem cell-derived cardiomyocytes.
\end{abstract}

\section{Keywords}

augment, right, ventricular, electrically, output, contractile, heart, polymers

Disciplines

Engineering | Physical Sciences and Mathematics

\section{Publication Details}

Ruhparwar, A., Piontek, P., Ungerer, M., Ghodsizad, A., Partovi, S., Foroughi, J., Szabo, G., Farag, M., Karck, M., Spinks, G. M. \& Kim, S. (2014). Electrically contractile polymers augment right ventricular output in the heart. Artificial Organs: replacement, recovery, and regeneration, 38 (12), 1034-1039.

\section{Authors}

Arjang Ruhparwar, Patricia Piontek, Matthias Ungerer, Ali Ghodsizad, Sasan Partovi, Javad Foroughi, Gabor Szabo, Mina Farag, Matthias Karck, Geoffrey M. Spinks, and Seon Jeong Kim 


\section{Electrically Contractile Polymers Augment Right Ventricular Output in the Heart}

\author{
*Arjang Ruhparwar, *Patricia Piontek, \\ *Matthias Ungerer, *Ali Ghodsizad, *Sasan Partovi, \\ †Javad Foroughi, *Gabor Szabo, *Mina Farag, \\ ${ }^{*}$ Matthias Karck, +Geoffrey M. Spinks, and \\ *Seon Jeong Kim \\ *Department of Cardiac Surgery, University \\ Hospital of Heidelberg, Heidelberg, Germany; \\ tARC Centre of Excellence for Electromaterials \\ Science, Intelligent Polymer Research Institute, \\ University of Wollongong, Wollongong, New South \\ Wales, Australia; and $¥$ Center for Bio-Artificial \\ Muscle, Department of Biomedical Engineering, \\ Hanyang University, Seoul, Korea
}

\begin{abstract}
Research into the development of artificial heart muscle has been limited to assembly of stem cellderived cardiomyocytes seeded around a matrix, while nonbiological approaches to tissue engineering have rarely been explored. The aim of the study was to apply electrically contractile polymer-based actuators as cardiomyoplasty for positive inotropic support of the right ventricle. Complex trilayer polypyrrole (PPy) bending polymers for high-speed applications were generated. Bending motion occurred directly as a result of electrochemically driven charging and discharging of the PPy layers. In a rat model $(n=5)$, strips of polymers $(3 \times 20$ $\mathrm{mm})$ were attached and wrapped around the right ventricle (RV). RV pressure was continuously monitored invasively by direct RV cannulation. Electrical activation occurred simultaneously with either diastole (in order to evaluate the polymer's stand-alone contraction capacity; group 1) or systole (group 2). In group 1, the pressure generation capacity of the polymers was measured by determining the area under the pressure curve (area under curve, AUC). In group 2, the RV pressure AUC was measured in complexes directly preceding those with polymer contraction and compared to RV pressure complexes with simultaneous polymer contraction. In group 1, the AUC generated by polymer contraction was $2768 \pm 875 \mathrm{U}$. In
\end{abstract}

doi:10.1111/aor.12300 group 2, concomitant polymer contraction significantly increased AUC compared with complexes without polymer support $(5987 \pm 1334 \mathrm{U}$ vs. $4318 \pm 691 \mathrm{U}, P \leq$ $0.01)$. Electrically contractile polymers are able to significantly augment right ventricular contraction. This approach may open new perspectives for myocardial tissue engineering, possibly in combination with fetal or embryonic stem cell-derived cardiomyocytes. Key Words: Right ventricle-Tissue engineering-Animal modelHeart failure approaches - Contractile polymers.

While heart transplantation, which creates a need for lifelong immunosuppression, remains the gold standard for the therapy of advanced and end-stage heart failure (1), research into regenerative therapy for partial or complete replacement of diseased myocardium by means of tissue engineering has led to remarkable results. Engineered heart tissue consisting of a collagen matrix and neonatal cardiomyocytes has been transplanted into small animals, resulting in improvement of ventricular function, and wholeheart tissue engineering has been performed by decellularization and reseeding of cells, essentially restoring myocardial function (2,3). Myocardial patches have also been created from embryonic stem cells (4). However, several challenges need to be overcome. Finding the optimal cell source remains a challenge. Fetal and neonatal cardiomyocytes are not available in large numbers, and embryonic stem cellderived or induced pluripotent stem cell-derived cells may cause teratoma (5). Additionally, nutrition of the constructs is dependent on diffusion, as no effective vascularization concept is available.

In analogy to cardiomyocytes, the application of polymers that contract upon low-voltage electrical stimulation could provide an additional tool for systolic augmentation of ventricular function. We have previously developed and characterized complex actuators consisting of trilayer polypyrrole (PPy) polymers that have the capacity for contraction and bending upon electrical stimulation at a low voltage of $0.5-1.0 \mathrm{~V}(6)$.

Due to the still limited force that can be generated, the low-pressure system of the circulation is the most likely target of myocardial tissue engineering approaches. The right ventricle, as an importantpart of the low-pressure system of the circulation, represents a suitable initial target for the study of myocardial regeneration by tissue engineering. 


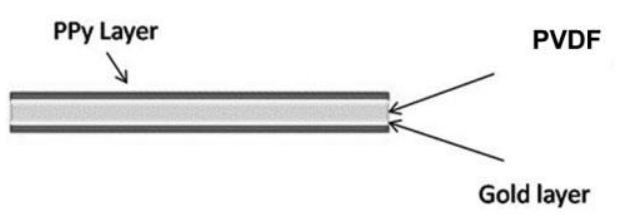

VDF

The aim of the present study was to apply electrically contractile polymer-based actuators as cardiomyoplasty for positive inotropic support of the right ventricle.

\section{MATERIALS AND METHODS}

\section{Synthesis of contracting actuators}

The contractile polymers were multilayer structures that have been described previously (6). Construction began by application of a thin, porous gold layer to both sides of a sheet of filter membrane material using sputter coating. The membrane consisted of polyvinylidene fluoride (PVDF) and was supplied as $110-\mu \mathrm{m}$-thick sheets with an average pore size of $0.45 \mu \mathrm{m}$. The gold coating was approximately 20-30 nm thick. A PPy coat was then applied on top of the gold layers to a thickness of $30 \mu \mathrm{m}$ on both sides of the membrane. PPy was deposited using an electrochemical polymerization process using a constant current density of $0.1 \mathrm{~mA} / \mathrm{cm}^{2}$ at $-31^{\circ} \mathrm{C}$ for 10 $\mathrm{h}$. The reaction solution contained $0.1 \mathrm{M}$ lithium trifluoromethanesulfonimide (LiTFSI) dissolved in propylene carbonate containing $1 \%$ (wt/wt) water. Before use, the bending actuators were thoroughly washed to remove any unreacted monomer and then soaked in propylene carbonate containing $0.1 \mathrm{M}$ LiTFSI. This solution was absorbed into the pores of the PVDF membrane and acted as the electrolyte needed for bending actuation, as described below. Strips of multilayer bending actuators were cut from larger sheets to approximately $30 \times 2 \mathrm{~mm}$. Electrical connections were made to both PPy layers at one end of each strip using platinum wire, and the entire assembly was sealed using a solvent-based acrylic polymer solution.

\section{Mechanism of action}

The bending motion occurs directly as a result of the electrochemically driven charging and discharging of the PPy layers. Charging the PPy by applying a positive voltage causes it to expand as it absorbs ions and solvent from the liquid electrolyte contained within the porous PVDF membrane. Shrinkage occurs at negative voltages due to the expulsion of ions and solvent as the PPy discharges. A bending motion is then produced when a potential difference is applied across the multilayer structure. A simple 1.5-V battery was used directly to power the actuator, with a polarity switch used to reverse the direction of bending. The structure of the multilayer actuators and principle of bending motion are illustrated in Fig. 1.

\section{Reagents and materials}

Pyrrole monomer was purchased from Merck KGaA (Darmstadt, Germany) and purified by distil- lation before use. PVDF membranes of $0.45 \mu \mathrm{m}$ pore size (Millipore, Billerica, MA, USA) were used as backing materials. LiTFSI was bought from 3M (St. Paul, MN, USA), and propylene carbonate was from SigmaAldrich (Castle Hill, Australia).

\section{Anesthesia and surgical technique}

During all experiments, the Principles of Laboratory Animal Care (NIH publication no. 86-23, revised 1985), as well as federal guidelines for the use of human tissue and the Animal Welfare Law of the state of Baden Württemberg, Germany, were followed. In a rat model $(n=5)$, anesthesia was performed by intraperitoneal injection of a combination of ketamine $(100 \mathrm{mg} / \mathrm{kg}$ body weight) and xylazine ( $3 \mathrm{mg} / \mathrm{kg}$ body weight), and the animals were put on a respirator (Small Animal Pressure Controlled Ventilator KTR-5 Dual Version, Hugo Sachs Elektronik-Harvard Apparatus GmbH, MarchHugstetten, Germany). The animals were heparinized using $400 \mathrm{IU} / \mathrm{kg}$ of heparin.

Via median sternotomy, strips of polymer were wrapped around and attached to the right ventricle. Strips of polymer were cut to the size of 3 $\mathrm{mm} \times 20 \mathrm{~mm}$ and wrapped semicircumferentially around the heart so that the right ventricle was covered. Attachment was performed using Bioglue Surgical Adhesive (Cryolife, Freiburg, Germany). Bioglue was identified as the best adhesive material after suturing with polypropylene, fibrin glue, and French glue did not produce reliable results. The free end of the polymer was attached to platinum 


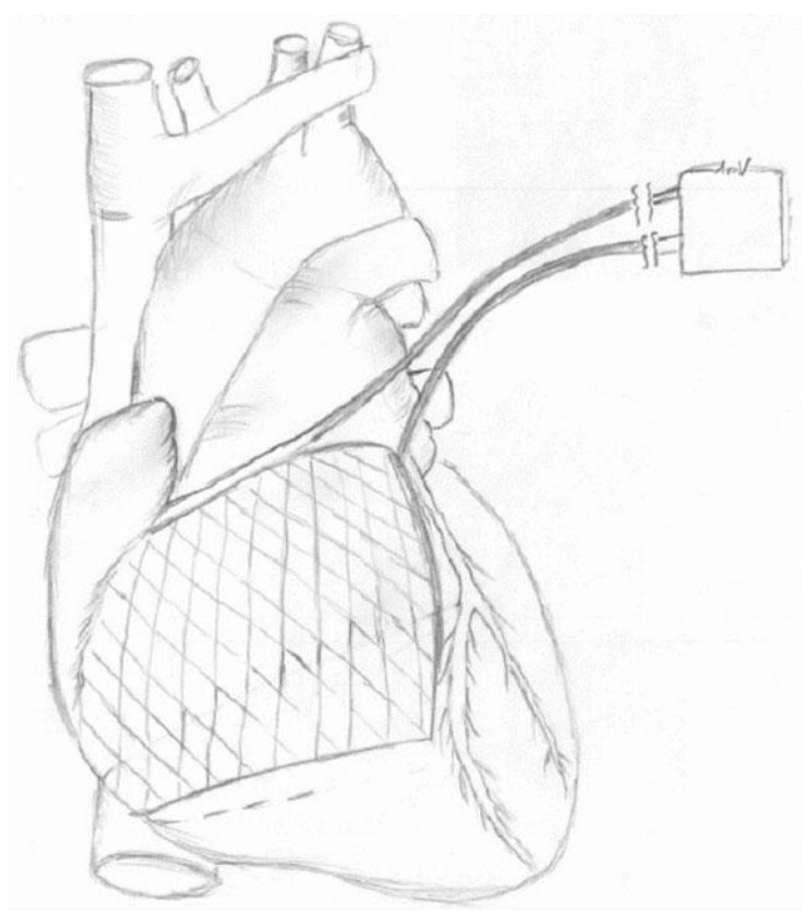

FIG. 2. Schematic of the setup of the electrically contractile polymer around the right ventricle.

electrodes that were attached to an electronic switch. Electric power was provided by an AA battery (Fig. 2).

\section{Hemodynamic monitoring}

Right ventricular (RV) pressure was continuously measured invasively by direct $R V$ cannulation with a 24G cannula. In analogy to the pulse contour cardiac output system (PiCCO; Pulsion Medical Systems AG, Munich, Germany), which calculates the stroke volume variation by continuous analysis of the arterial pulse contour (7), the right ventricular area under the pressure-time curve was measured in arbitrary units $(\mathrm{U})$ as a correlate to stroke volume. The artifact-free complexes were chosen for analysis. This $\mathrm{RV}$-pressure area under the curve (AUC) was measured in complexes directly preceding those with polymer contraction and compared with RVpressure complexes with polymer contraction during systole or diastole. Monitoring was performed using an APT300 blood pressure transducer, Plugsys Mini Case Type 609 Amplifier, and HSE-BDAS W software (all Hugo Sachs Elektronik-Harvard Apparatus $\mathrm{GmbH}$ ) and recorded on a computer (Toshiba Satellite Pro L300, Neuss, Germany).

\section{Polymer activation}

Electrical activation $(0.5 \mathrm{~V})$ occurred simultaneously with diastole (immediately after the end of sys- tolic contraction) in order to evaluate the polymer's stand-alone contraction capacity (group 1) or at the beginning of systolic contraction in order to evaluate systolic augmentation capacity for the right ventricle (group 2). The experimental setup is depicted in Fig. 3.

\section{Statistical analysis}

The statistical analysis was performed with SPSS version 20 (SPSS, Inc., Chicago, IL, USA). The relative change compared with the AUC before polymer contraction was expressed as a percentage. Variables were expressed as mean \pm standard deviation. The AUCs with and without polymer contraction were compared using the paired $t$-test after testing for normality of distribution and application of the Wilcoxon signed rank test. The relationships between the variables were further characterized using Pearson's correlation analysis and regression analysis. The results were considered to be significant if $P$ was smaller than 0.05 .

\section{RESULTS}

\section{Successful establishment of animal model}

Seventy-two animals were required for success- ful implementation of the animal model. Creation of a polymer-myocardium interface with $1: 1$ force transduction from polymer to the right ventricle was the greatest challenge in this model. After several attempts using fibrin glue (Baxter $\mathrm{GmbH}$, Unterschleissheim, Germany), gelatin-resorcinol-formalin glue (Cardial, Technopole, Sainte-Etienne, France) and polypropylene sutures, Bioglue Surgical Adhesive (Cryolife) turned out to be the only means to achieve high mechanical stability in this model.

\section{Stand-alone pressure generation capacity of the polymer}

In group 1, where polymer contraction was activated during diastole, the AUC generated by polymer contraction was $2768 \pm 875 \mathrm{U}$ (Fig. 4C).

\section{Improvement of right ventricular function by polymer contraction}

Concomitant polymer contraction significantly increased AUC compared with complexes without polymer support. Figures $4-6$ show the final results. Figure 5 shows that an increase in the AUC was achieved following polymer contraction in all 63 measurements. The relative increase in the AUC before polymer contraction $(4318.33 \pm 691.34 \mathrm{U})$ compared with the AUC after polymer contrac- tion $(5987.22 \pm 1334.96 \mathrm{U})$ averaged $38 \%$. The two 


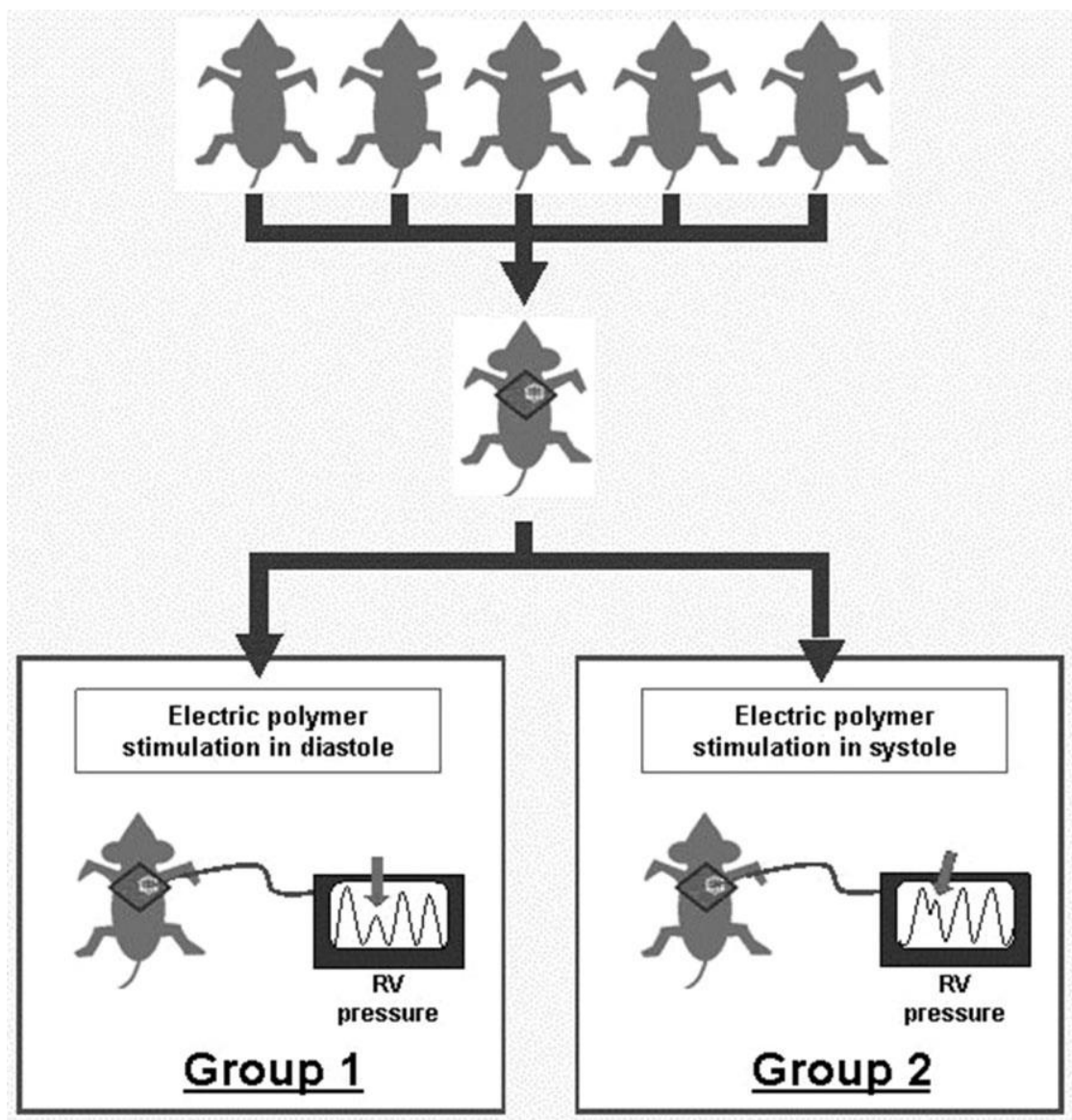

FIG. 3. Successful establishment of animal model. Animals were thoracotomized, and invasive right ventricular pressure was recorded. Arrows show changes of RV pressure by electrical polymer activation.

measurement series were found to be statistically different using the parametric paired $t$-test $(P<0.01)$ and the nonparametric Wilcoxon signed-rank test $(P$ $<0.01)$. We found a significant correlation between both series using the Pearson coefficient $(P<0.01)$. The relative change was compared with the AUC before the intervention in Fig. 6. The regression analysis found a quadratic relationship $(P<0.01)$ between these variables, suggesting that the observed relative changes in AUC were greatest in hearts with moderate preintervention contractility.

\section{DISCUSSION}

A rat model involving stable attachment of electrically contractile polymers around the right ventricle and invasive recording of right ventricular pressure and stroke volume was successfully established. Continuous invasive measurement of right ventricular pressure without and with electrical activation of contractile polymers revealed a significant increase in AUC and thereby right ventricular stroke volume upon electrical activation and contraction of the polymer. In analogy to the left ventricle in other studies, the greatest benefit was observed in animals in which right ventricular stroke volume was moderate due to loss of blood, mechanical manipulation of the right ventricle, or the effect of the glue that was used for the attachment of the polymer during the experiment.

Wrapping of engineered structures around the heart for support of myocardial function has been performed with noncontractile devices such as Acorn Cardiovascular's CorCap polyester mesh (CSD; Acorn Cardiovascular, Inc., St. Paul, MN, USA), resulting in reduced stress on myocardial fibers (8). The use of contractile elements around the heart should have a more synergistic effect on myocardial function. As most tissue engineering approaches for the augmentation of myocardial function will most likely be performed on the right ventricle due to the limited power these constructs can generate, a con- ceivable clinical application would be in the Fontan circulation, as moderate increase in right atrial pres- sure can significantly improve pulmonary artery flow and lung perfusion. Voss et al. were able to achieve this goal by dynamic cardiomyoplasty using skeletal muscle in a canine model (9). Tenfold magnification 

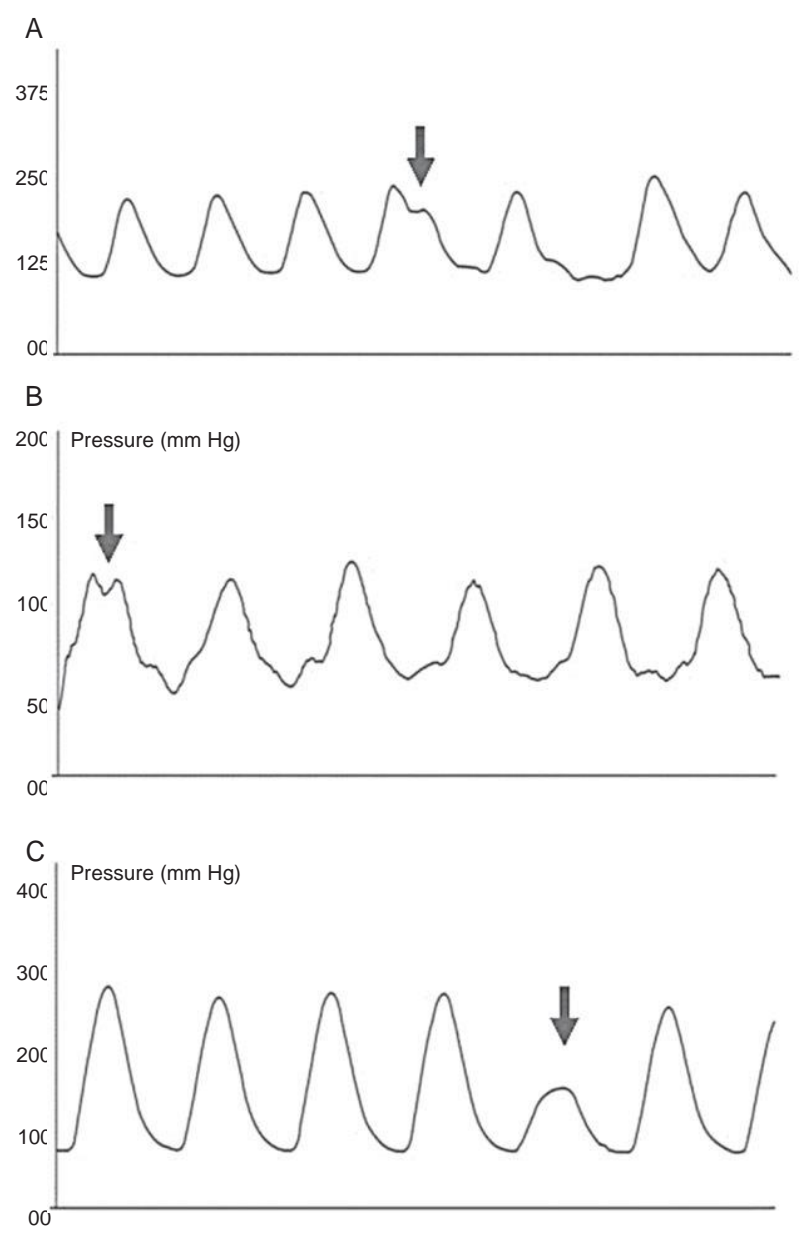

FIG. 4. Concomitant polymer contraction significantly increased AUC compared with complexes without polymer support. Representative invasive measurement results of right ventricular pressure during systole show augmentation of RV pressure during systole by polymer contraction (red arrows, A and B) and standalone capacity for pressure generation of the polymer by contraction during diastole (red arrow, C).

of the polymers shows a similar gross structure as present in the myocardium itself, showing contractile fibers with large spaces between them, possibly allowing for additional seeding of cardiomyocytes onto the polymer with the intention for synergistic generation of force in future studies (Supporting Information Fig. S1). Another challenge will be the elimination of fatigue and increase of durability, which is at this point limited to several hours but is already increasing with new-generation polymers (articles in preparation). Moreover, although proof of concept with regard to augmentation of cardiac output was achieved, it remains to be demonstrated that contractile polymers are as effective in animal models with RV failure as in our experimental setting, where, as a first step, animals with healthy right ventricles were chosen.

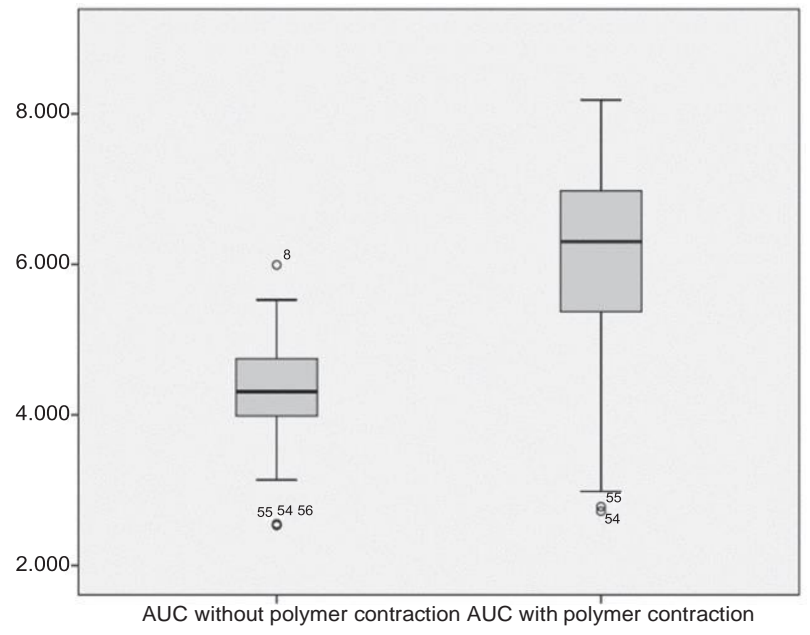

FIG. 5. Boxplot showing a direct comparison of medians and data distribution before and after polymer contraction in group 2 . Concomitant polymer contraction significantly increased AUC compared with complexes without polymer support.

We made compromises in order to show proof of concept of increase of RV output by contractile polymers. However, several limitations of our study design need to be addressed.

The maximum contraction rate that can be achieved by the polymers is $60 / \mathrm{min}$. Therefore RV augmentation could not be performed for every cardiac cycle due to the high heart rate in this animal model. We avoided administration of negative chronotropic drugs as they may also have a negative inotropic effect.

Additionally, titration of adenosine and shorteffect beta-blockers such as esmolol turned out to be

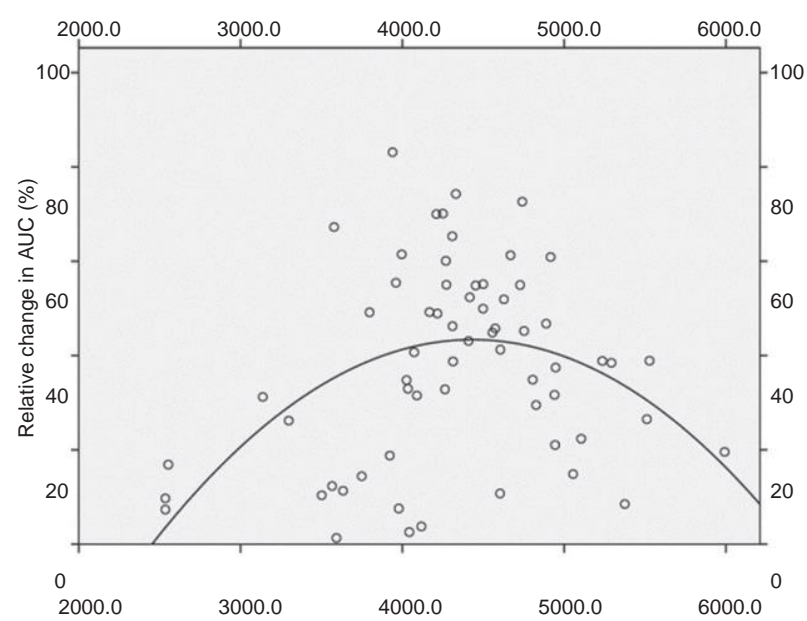

FIG. 6. Scatterplot comparing the AUC without polymer contraction with the relative increase in AUC following polymer contraction in group 2 . The quadratic distribution $(P<0.01)$ suggests that rat hearts with moderate preinterventional AUC received the greatest benefit from polymer contraction. 
unreliable with regard to the duration of bradycardia and hemodynamic instability. However, with technical progress, higher contraction rates, and larger sizes of the polymers, large-animal experiments will be feasible. In the future, we plan to document RV output in such an experiment, using continuous pressure-volume loops in order to bolster our results.

\section{CONCLUSION}

Electrically contractile polymers are able to significantly augment right ventricular contraction. Our approach may open new perspectives for myocardial tissue engineering, possibly in combination with cardiomyocytes.

\section{Conflict of Interest: None.}

\section{REFERENCES}

1. Kittleson MM, Kobashigawa JA. Management of the ACC/ AHA Stage D patient: cardiac transplantation. Cardiol Clin 2014;32:95-112.

2. Zimmermann WH, Melnychenko I, Wasmeier G, et al. Engineered heart tissue grafts improve systolic and diastolic function in infarcted rat hearts. Nat Med 2006;12:452-8.
3. Ott HC, Matthiesen TS, Goh SK, et al. Perfusion-decellularized matrix: using nature's platform to engineer a bioartificial heart. Nat Med 2008;14:213-21.

4. Hayashi T, Misawa H, Nakahara $\mathrm{H}$, et al. Transplantation of osteogenically differentiated mouse iPS cells for bone repair. Cell Transplant 2012;21:591-600.

5. Stevens KR, Pabon L, Muskheli V, Murry CE. Scaffold-free human cardiac tissue patch created from embryonic stem cells. Tissue Eng Part A 2009;15:1211-22.

6. Wu Y, Alici G, Spinks GM, Wallace GG. Fast trilayer polypyrrole bending actuators for high speed applications. Synth Met 2006;156:1017-22.

7. Combes A, Berneau JB, Luyt CE, Trouillet JL. Estimation of left ventricular systolic function by single transpulmonary thermodilution. Intensive Care Med 2004;30:1377-83.

8. Wenk JF, Ge L, Zhang Z, et al. Biventricular finite element modeling of the Acorn CorCap Cardiac Support Device on a failing heart. Ann Thorac Surg 2013;95:2022-7.

9. Voss B, Sack FU, Saggau W, Hagl S, Lange R. Atrial cardiomyoplasty in Fontan circulation. Eur J Cardiothorac Surg 2002;21:780-6.

\section{SUPPORTING INFORMATION}

Additional Supporting Information may be found in the online version of this article at the publisher's web-site:

Fig. S1. Tenfold magnification of the contracting polymer reveals spaces between the fibers, possibly allowing for seeding of cardiomyocytes onto the polymer, with the potential for synergistic effects. 